\title{
Cortical Map Plasticity as a Function of Vagus Nerve Stimulation Rate
}

\author{
E.P. Buell ${ }^{1,2}$, K.W. Loerwald ${ }^{1,2}$, C.T. Engineer ${ }^{1,2}$, M.S. Borland ${ }^{1,2}$, J.M. Buell ${ }^{1}$, C.A. Kelly ${ }^{2}$, I.I. \\ Khan $^{2}$, S.A Hays ${ }^{1,3}$, and M.P. Kilgard ${ }^{1,2}$ \\ ${ }^{1}$ Texas Biomedical Device Center, Richardson, TX 75080 \\ ${ }^{2}$ The University of Texas at Dallas, School of Behavioral Brain Sciences, 800 West Campbell \\ Road, GR 41, Richardson, TX 75080-3021 \\ ${ }^{3}$ The University of Texas at Dallas, Erik Jonsson School of Engineering and Computer Science, \\ BSB 11; Richardson, TX 75080
}

\begin{abstract}
Background-Repeatedly pairing a brief train of vagus nerve stimulation (VNS) with an external event can reorganize the sensory or motor cortex. A $30 \mathrm{~Hz}$ train of sixteen VNS pulses paired with a tone significantly increases the number of neurons in primary auditory cortex (A1) that respond to tones near the paired tone frequency. The effective range of VNS pulse rates for driving cortical map plasticity has not been defined.
\end{abstract}

Objective/Hypothesis-This project investigated the effects of VNS rate on cortical plasticity. We expected that VNS pulse rate would affect the degree of plasticity caused by VNS-tone pairing.

Methods-Rats received sixteen pulses of VNS delivered at a low $(7.5 \mathrm{~Hz})$, moderate $(30 \mathrm{~Hz})$, or high $(120 \mathrm{~Hz})$ rate paired with $9 \mathrm{kHz}$ tones 300 times per day over a 20 day period.

Results-More A1 neurons responded to the paired tone frequency in rats from the moderate rate VNS group compared to naïve controls. The response strength was also increased in these rats. In contrast, rats that received high or low rate VNS failed to exhibit a significant increase in the number of neurons tuned to sounds near $9 \mathrm{kHz}$.

Conclusion-Our results demonstrate that the degree of cortical plasticity caused by VNS-tone pairing is an inverted-U function of VNS pulse rate. The high temporal precision of VNS-tone pairing protocols helps to explain the cellular mechanisms responsible for the beneficial effects of precisely timed VNS during restoration of sensory or motor function.

\section{Keywords}

plasticity; auditory cortex; vagal nerve stimulation; inverted-u function; frequency

*Corresponding author information: The University of Texas at Dallas, School of Behavioral and Brain Sciences, 800 West Campbell Road, BSB11, Richardson, TX 75080-3021, ebuell@utdallas.edu, Tel: 1-469-939-0422.

Publisher's Disclaimer: Disclaimer

Any opinions, findings, and conclusions or recommendations expressed in this publication are those of the author(s) and do not necessarily reflect the views of the Defense Advanced Research Projects Agency (DARPA) Biological Technologies Office (BTO). 


\section{Introduction}

Repeatedly pairing VNS with a sensory or motor event drives robust experience-dependent plasticity in the auditory and motor cortices [1-4]. Pairing brief bursts of VNS with a movement expands the area associated with that movement in the motor cortex and increases the synaptic connectivity from the associated muscles to motor cortex [5-7]. VNS paired with a pure tone increases the number of cortical neurons that respond to the paired tone $[1,4]$. VNS-directed neural plasticity is being developed to treat a variety of disorders, including tinnitus and stroke [3,4,8-15]. Optimization of VNS parameters is a critical next step in the development of these therapies. Parameter optimization may also help to elucidate the neural mechanisms that allow brief bursts of VNS to direct plasticity that is long lasting and specific to the experience paired with VNS.

Previous studies indicate that VNS parameters, such as the interval between stimulation events, stimulation intensity and stimulation rate, can influence the effectiveness of VNS in producing cortical plasticity $[1,16,17]$. The stimulation intensity paired with a tone drives cortical plasticity as an inverted-U function [1]. Moderate intensity (0.4-0.8 mA) VNS drives plasticity, while high intensity (1.2-1.6 mA) VNS does not. VNS-tone pairing produces maximal plasticity when the interval between events is greater than thirty seconds [16]. Increasing VNS pulse width enhances plasticity when low currents are used [17]. These studies support the notion that the timing and intensity of stimulation influence the degree of VNS-dependent plasticity, but the effect of VNS rate on cortical plasticity remain unknown.

Changing VNS rate has significant effects on seizure suppression and activity patterns in brainstem nuclei [18]. VNS drives robust activity in the locus coeruleus (LC) across a wide range of stimulation rates [19]. In contrast, activity in the dorsal raphe increases as an inverted-U function of VNS rate [20]. Some studies report that moderate VNS rates are most effective in suppressing seizures [21,22], while others report that higher stimulation rates are most effective [23]. Together, these studies indicate that VNS pulse frequency affects engagement of neuromodulatory networks and may impact the clinical efficacy of VNS, pointing to the relevance of optimizing this parameter.

The purpose of this study was to evaluate whether delivering VNS-tone therapy using different VNS rates changes the degree of plasticity in the auditory cortex. We tested the hypothesis that stimulation rate would affect the degree of VNS-dependent plasticity in primary auditory cortex by evaluating cortical changes after pairing low $(7.5 \mathrm{~Hz})$, moderate $(30 \mathrm{~Hz})$, or high $(120 \mathrm{~Hz})$ rate VNS with a $9 \mathrm{kHz}$ tone. Understanding how brain plasticity is affected by the rate of VNS paired with an event is critical to identify the optimal stimulation settings for clinical applications.

\section{Methods}

Data from forty-one adult female Sprague-Dawley rats (250-400 g) was analyzed in this experiment. Seventy-five rats were implanted with vagus nerve cuff stimulators, as in our previous studies $[1,4,5,24]$. Animals were removed from this study due to surgical deaths 
$(n=16)$, head cap failure ( $n=7)$, or a broken VNS lead ( $n=10$, confirmed by an inability to reduce blood oxygen saturation in anesthetized rats via continuous VNS) [25]. All animals included in the analysis demonstrated a drop in blood oxygen saturation during the terminal surgery procedure which indicates a functional cuff. Twelve rats served as naïve controls and were not implanted. Rats were interleaved and randomly assigned to receive low rate (7.5 $\mathrm{Hz})$ VNS $(\mathrm{n}=9)$, moderate rate $(30 \mathrm{~Hz})$ VNS group $(\mathrm{n}=10)$, or high rate $(120 \mathrm{~Hz}) \mathrm{VNS}$ group $(\mathrm{n}=10)$. All rats were housed in a 12:12 hour reversed light-dark cycle. All handling, housing, stimulation, and surgical procedures were approved by The University of Texas at Dallas Institutional Animal Care and Use Committee and by the Animal Care and Use Review Office of the United States Army Medical Research and Materiel Command Office of Research Protections.

\section{Vagus nerve surgery}

Animals were anesthetized using ketamine hydrochloride $(80 \mathrm{mg} / \mathrm{kg}$, intraperitoneal (IP) injection) and xylazine (10 mg/kg IP). Animals were given supplemental doses as needed. A Ringer's lactate and dextrose solution was given to the rats to prevent dehydration. Cefotaxime sodium $(2 \times 10 \mathrm{mg}$, subcutaneous (SC) injection) solution was administered to animals after the surgery to prevent infection. All animals were implanted with a skull mounted connector. Marcaine $(1 \mathrm{~mL}, \mathrm{SC})$ was injected into the scalp at the incision site. Bregma and lambda landmarks on the skull were exposed by an incision of the scalp running anterior to posterior. Bone screws were placed near the bregma suture, near the sagittal suture, near the lambda suture and over the cerebellum. The connector headcap was secured to the screws with acrylic. A custom made cuff electrode was placed around the left vagus nerve as in previous studies [1,4,5,24]. Because the right vagus nerve innervates the sinoatrial node, only the left vagus nerve was implanted to avoid cardiac complications [26]. Brain activation following left VNS is bilateral [27].

After lidocaine (2\%, $0.5 \mathrm{~mL} \mathrm{SC})$ was injected in the neck at the incision site, the vagus nerve was exposed through an incision and blunt dissection of the neck. The cuff electrode was placed around the nerve. Two Teflon coated multi stranded platinum iridium wire leads from the electrode were run subcutaneously between the eye and the ear, and connected to the headcap on top of the skull. The headcap and cuff pins were secured with acrylic. After connection, confirmation of cuff function was obtained by observation of a blood oxygen drop while stimulating the nerve (A-M Systems isolated pulse stimulator model 2100). The animal's neck was sutured closed, and a topical antibiotic cream was applied to the neck and head. Animals were given amoxicillin $(5 \mathrm{mg})$ and carprofen $(1 \mathrm{mg})$ for 2 days after surgery to prevent infection and facilitate recovery.

\section{Vagus nerve stimulation}

After 5-7 days of recovery from surgery, the rats received VNS paired with a $9 \mathrm{kHz}$ tone 300 times per day for 20 days, as in previous studies [1,4,17]. Animals were placed in a 25 $\mathrm{cm} \times 25 \mathrm{~cm} \times 25 \mathrm{~cm}$ wire cage within a $50 \mathrm{~cm} \times 60 \mathrm{~cm} \times 70 \mathrm{~cm}$ chamber lined with acoustic insulating foam. A speaker hanging above the cage presented a $9 \mathrm{kHz}$ tone at $50 \mathrm{~dB}$ SPL paired $150 \mathrm{~ms}$ after the onset of VNS. VNS was delivered using $100 \mu \mathrm{s}$ charge balanced biphasic pulses. Experimental groups received 16 pulses of VNS delivered at rates of $7.5 \mathrm{~Hz}$, 
$30 \mathrm{~Hz}$, or $120 \mathrm{~Hz}$, such that the pulses are distributed over 2000, 500, or $125 \mathrm{~ms}$ (Figure 1). VNS intensity was $0.8 \mathrm{~mA}$ for all experimental groups. Stimulation was delivered $50 \%$ of the time every 15 seconds, such that the average interval between stimulations was 30 seconds. The impedance of the cuff electrode was checked daily and only animals with measured cuff impedances between 1 and $10 \mathrm{k} \Omega$ remained in the study.

\section{Auditory cortex recordings}

Using standard procedures, multi-unit responses were collected from the auditory cortex twenty-four hours after the final VNS-tone pairing session [1,4,16,28]. Rats were anesthetized with sodium pentobarbital $(50 \mathrm{mg} / \mathrm{kg}$ ) and anesthesia was maintained using supplemental doses of diluted pentobarbital by evaluating anesthesia levels between cortex recordings every 30-60 minutes $(0.2-0.4 \mathrm{ml}, 8 \mathrm{mg} / \mathrm{ml})$. One $\mathrm{mL}$ of a one to one ratio of dextrose (5\%) and standard Ringer's lactate solution was administered between every recording to prevent dehydration. To minimize respiratory problems under pentobarbital, a tracheotomy was performed. In addition, cerebral edema was minimized by opening a cisternal drain. A craniotomy exposed the right primary auditory cortex. A durotomy over this exposed section of cortex further exposed the cortex and allowed electrodes to be placed easily. A thin film of silicone oil was placed over the exposed cortex to maintain hydration. Four parylene coated tungsten microelectrodes (1.5-2.5 M 2 , FHC) were lowered to layer IV/V of the primary auditory cortex. Pure tones were delivered from a speaker positioned 10 $\mathrm{cm}$ from the animal's left ear in a foam-shielded double-walled sound-attenuated chamber. Neuronal responses were recorded using Brainware software (TDT). Recording sites were tracked using a photo of the cortex in Canvas 16 software. Various tones were presented at 81 logarithmically spaced frequencies spanning $1-32 \mathrm{kHz}$ in 0.0625 octave steps at 16 intensities from 0 to $75 \mathrm{~dB}$ SPL in $5 \mathrm{~dB}$ steps. These tones were used to determine auditory tuning curves at each of the evaluated sites (Figure 2). Tones were presented every $500 \mathrm{~ms}$ and randomly interleaved. For each auditory map, experimenters were blind to the animal's stimulation parameters. At the conclusion of the map, cuff function was confirmed by testing an oxygen saturation drop in response to 10 seconds of continuous vagus nerve stimulation. If an $\mathrm{O}_{2}$ saturation drop could not be confirmed, the animal was removed from the study.

\section{Data analysis}

All groups were analyzed using an automated MATLAB program. This program determines receptive fields based on the characteristics of responses at each site as determined by previous work [28]. The characteristic frequency $(\mathrm{CF})$ was defined at the frequency at which the lowest intensity evokes a response at a particular site. Response threshold was defined as the lowest intensity capable of evoking a response (Figure 2). Spontaneous firing rate was the rate of firing evoked across all tone frequencies when presented at an amplitude of $0 \mathrm{~dB}$. The time it takes for maximum neural responses to occur was defined as the peak latency. For percentage of cortex responding analysis, experimental and control groups were compared using a one-way ANOVA with post-hoc Bonferroni correction for multiple comparisons. For response strength analysis, a mixed-effects model using SPSS software was used to account for the different number of sites recorded for each animal. The fixed factor was the experimental group and the random factor was the individual animals. Simple 
contrast analysis was used to determine whether there were statistically significant differences in response strength after VNS-tone pairing.

\section{Results}

\section{VNS-tone pairing shifts $A 1$ receptive fields if moderate VNS is used}

Plasticity in the auditory cortex was evaluated by quantifying the percentage of A1 neurons that responded to individual tones across a wide range of frequency and intensity. In experimentally naïve control rats, approximately $40 \%$ of A1 neurons responded to $50 \mathrm{~dB}$ SPL 8-16 kHz tones. VNS-tone pairing significantly altered the percentage of A1 neurons responding to the paired tone frequency $(\mathrm{F}(3,37)=11, \mathrm{p}=0.00003)$. After pairing $9 \mathrm{kHz}$ tones with moderate rate VNS, significantly more A1 neurons responded to these tones (Figure 3, $\mathrm{p}=0.001$ ). This observation represents the fifth independent replication of the finding that pairing VNS with tones alters the organization of auditory cortex [1,4,16,17]. Pairing the same tones with an identical amount of VNS (i.e. sixteen $100 \mu$ s pulses at 0.8 $\mathrm{mA}$ ) delivered at a lower or higher rate did not significantly alter frequency tuning compared to control rats $(p>0.05)$. These findings demonstrate that cortical plasticity is an inverted-U function of the VNS rate used during VNS-tone pairing.

Figure 4 shows the proportion of A1 neurons that responded to each tone frequency and intensity combination. Across all groups, more A1 neurons were activated as sound intensity was increased (Figure 4, A-D). The only group with a consistently expanded response to tones was the moderate VNS group, which exhibited a significant increase in the number of neurons tuned to tones with a frequency between 8 and $16 \mathrm{kHz}$ and intensities above $20 \mathrm{~dB}$ SPL. This difference is best seen by subtracting the proportion of A1 neurons that responded in experimentally naïve control rats from the proportion of A1 that responded in the moderate VNS group (Figure 4F). Rats from the low rate VNS group and the high rate VNS group did not exhibit a significant increase in the number of A1 neurons that responded to any tone when compared to control rats $(p>0.05$, Figures $4 \mathrm{E}$ and $4 \mathrm{G})$.

\section{VNS-tone pairing alters the A1 response strength if moderate VNS is used}

VNS-tone pairing significantly altered the number of action potentials evoked in A1 in response to frequencies near the paired tone $(F(3,34.95)=6.2, p=0.002)$. Tones between $8-16 \mathrm{kHz}$ evoked significantly more action potentials in the moderate rate VNS group compared to the control group ( $0.93 \pm 0.08$ spikes vs. $0.62 \pm 0.07$ spikes, $p=0.01$, Figure 5). In contrast, the low rate VNS group and the high rate VNS group response strength to these tones were not significantly different than naïve control rats ( $p>0.05$, Figure 5). There was a significant interaction between experimental group and tone intensity $(F(45,33930.85)=$ $2.83, \mathrm{p}<0.0001$, Figure 6). VNS-tone pairing produced a $50 \%$ increase in the A1 response to the paired tone frequency across a wide range of tone intensities (Figure 6) when moderate rate VNS $(p=0.03)$ was used and failed to alter the cortical response when higher or lower VNS rates were used ( $p>0.05)$. This observation is consistent with the inverted-U function for receptive field plasticity. 
No significant changes in response threshold $(\mathrm{F}(3,40.43)=1.24, \mathrm{p}=0.31)$, spontaneous firing rate $(\mathrm{F}(3,34.12)=0.21, \mathrm{p}=0.89)$, bandwidth $(\mathrm{F}(3,37.66)=2.47, \mathrm{p}=0.08)$, or response peak latency $(\mathrm{F}(3,44.82)=0.22, \mathrm{p}=0.88)$ were observed in any experimental group compared to the control group.

\section{Discussion}

More than a dozen studies have confirmed that repeatedly pairing VNS with an auditory or motor event can drive highly specific and long-lasting plasticity in primary auditory or motor cortex, respectively $[1,2,4-6,11,16,17,24,29-34]$. Since VNS-directed neural plasticity appears to enhance recovery of auditory and motor function following injury $[3,4,6,8,12,14,15,34]$, it is critical to understand the optimal conditions to drive therapeutic plasticity. Previous papers have systematically evaluated how the stimulation intensity, spacing between pairings, pulse width, and number of VNS-tone pairings influence the degree of neural plasticity $[1,16,17]$. This paper systematically explored the influence of VNS rate and provides the first demonstration that only moderate rate VNS-tone pairing produces plasticity. When VNS is delivered at a fast or slow rate, VNS-tone pairing fails to produce significant cortical map plasticity. This finding helps to clarify potential mechanisms through which VNS-tone pairing drives neural plasticity and suggests that future studies of other VNS rates near $30 \mathrm{~Hz}$ are needed to determine whether $30 \mathrm{~Hz}$ is the most effective VNS rate for directing neural plasticity.

Multiple modulatory neurotransmitters influence plasticity, and phasic and tonic levels of these neurotransmitters can have very different effects [35-37]. Numerous studies have documented that VNS alters cortical function through activation of the cholinergic nucleus basalis (NB) and noradrenergic locus coeruleus (LC) [19,29,38-42]. VNS produces robust activation of LC neurons [19]. LC activity releases norepinephrine, which is known to modulate synaptic plasticity [35]. Sixteen pulses of VNS produce the same number of LC action potentials regardless of whether the rate is $7.5,30$, or $120 \mathrm{~Hz}$. However, the temporal pattern evoked is quite different, because the same number of action potentials is spread out over 2000,500 , or $125 \mathrm{~ms}$. It is possible that the inverted-U function of VNS rate on cortical plasticity results from inadequate norepinephrine concentration to activate the high affinity alpha receptors at low rates and overly high norepinephrine concentration that activates low affinity beta receptors at high rates [43]. Pharmacology studies will be needed to test this hypothesis.

A second possible explanation for the inverted-U function of VNS rate on plasticity is based on the observation that neurons in the dorsal raphe nucleus (DRN) are selectively activated by moderate VNS rates. VNS increases DRN firing as an inverted-U function of VNS rate [20]. Since serotonin is known to modulate synaptic plasticity, such changes in DRN activity could contribute to our results [35].

Previous studies revealed that VNS effects on memory, cortical plasticity, hippocampal LTP, and DRN activity are an inverted-U function of current intensity [1,20,44-46]. These effects of current likely arise in the periphery because moderate currents recruit large diameter vagus nerve fibers without recruiting small diameter nerve fibers, which limit plasticity via 
an unknown mechanism [47]. In contrast, the inverted-U relationship between plasticity and VNS rate must result from a central action because vagus nerve fibers can fire at rates up to $150 \mathrm{~Hz}$. Thus, VNS current and rate both appear to influence plasticity as inverted-U functions, but appear to result from distinctly different mechanisms. Given the richness of synaptic plasticity rules, future studies are needed to empirically determine how VNS rate, VNS duration, and relative VNS-tone timing influence neural plasticity. It remains to be seen whether other VNS paradigms are more effective at directing neural plasticity.

Understanding how the VNS parameter space influences plasticity may help to elucidate the cellular mechanisms that influence cortical plasticity by providing a clinically viable method to deliver brief bursts of neuromodulator release $[11,48]$. These findings may facilitate determination of optimal VNS parameters for clinical application. Recent clinical studies of targeted VNS pairing plasticity therapies for tinnitus and stroke suggest non-responder rates between 40 and $50 \%$. These therapies would be more effective if parameter optimization could be used to reduce the number of non-responders [14,49].

In summary, the results in this experiment demonstrate that the rate of tone-paired VNS influences the extent of cortical change. Repeatedly pairing sixteen $0.8 \mathrm{~mA}$ pulses of VNS delivered at $30 \mathrm{~Hz}$ with a tone effectively drives map expansion in the auditory cortex. The same amount of VNS fails to drive plasticity when delivered at a faster $(120 \mathrm{~Hz})$ or slower $(7.5 \mathrm{~Hz})$ rate. This inverted $\mathrm{U}$-function of rate likely arises from a central mechanism regulating synaptic plasticity. A better understanding of how VNS regulates these mechanisms could potentially lead to the development of novel adjuvant therapies to improve recovery from sensory, emotional, or motor disorders $[3,4,7,11,13-15,48,50]$.

\section{Acknowledgements}

We would like to thank Alan Carroll, Emily Jensen, Madelyne Frech, Natasha Houshmand, Pryanka Sharma, Shen Xian, Mark Lane, Aisha Khan, Lena Sadler, Collin Chandler, Eric Meyers, Camilo Sanchez, Jayant Kurvari, Jordan Chen, Daniel Kaminski, Son Pham, and Samuel Yang for their contributions to this project.

Financial Disclosure

This work was sponsored by the Defense Advanced Research Projects Agency (DARPA) Biological Technologies Office (BTO) ElectRx and TNT programs under the auspices of Dr. Doug Weber, Eric VanGieson, and Tristan McClure-Begley through the Space and Naval Warfare Systems Center, Pacific Grant/Contract Nos. N66001-15-2-4057 and N66001-17-2-4011. MPK is a consultant for, and has a financial interest in, MicroTransponder, Inc., which is developing therapies using VNS. EPB, KWL, CTE, MSB, JMB, CAC, IIK, and SAH report no biomedical financial interests or potential conflicts of interest.

\section{References}

[1]. Borland MS, Vrana WA, Moreno NA, Fogarty EA, Buell EP, Sharma P, et al. Cortical Map Plasticity as a Function of Vagus Nerve Stimulation Intensity. Brain Stimul 2016;9:117-23. 10.1016/j.brs.2015.08.018. [PubMed: 26460200]

[2]. Engineer CT, Engineer ND, Riley JR, Seale JD, Kilgard MP. Brain Stimulation Pairing Speech Sounds With Vagus Nerve Stimulation Drives Stimulus-speci fi c Cortical Plasticity. Brain Stimul 2015;8:637-44. 10.1016/j.brs.2015.01.408. [PubMed: 25732785]

[3]. Hays SA, Khodaparast N, Hulsey DR, Ruiz A, Sloan AM, Ii RLR, et al. Training Improves Functional Recovery After Intracerebral Hemorrhage. Stroke 2014;45:97-100. 10.1161/ STROKEAHA.114.006654. 
[4]. Engineer N, Riley JR, Seale JD, Vrana WA, Shetake JA, Sudanagunta SP, et al. Reversing pathological neural activity using targeted plasticity. Neurosurgery 2011;68:101-4. 10.1227/01.neu.0000395792.97271.ae.

[5]. Porter BA, Khodaparast N, Fayyaz T, Cheung RJ, Ahmed SS, Vrana WA, et al. Repeatedly Pairing Vagus Nerve Stimulation with a Movement Reorganizes Primary Motor Cortex. Cereb Cortex 2012;22:2365-74. 10.1093/cercor/bhr316. [PubMed: 22079923]

[6]. Meyers EC, Solorzano BR, James J, Ganzer PD, Lai ES, Rennaker RL, et al. Vagus Nerve Stimulation Enhances Stable Plasticity and Generalization of Stroke Recovery. Stroke 2018:10.1161/STROKEAHA.117.019202.

[7]. Ganzer PD, Darrow MJ, Meyers EC, Solorzano BR, Ruiz AD, Robertson NM, et al. Closed-loop Neuroprosthesis Restores Network Connectivity and Motor 2 Control after Spinal Cord Injury. Elife n.d

[8]. Dawson J, Pierce D, Dixit A, Kimberley TJ, Robertson M, Tarver B, et al. Safety, Feasibility, and Efficacy of Vagus Nerve Stimulation Paired With Upper-Limb Rehabilitation After Ischemic Stroke. Stroke 2015;47:143-50. 10.1161/STROKEAHA.115.010477. [PubMed: 26645257]

[9]. De Ridder D, Vanneste S. Placebo-Controlled Vagus Nerve Stimulation Paired with Tones in a Patient With Refractory Tinnitus: A Case Report. Otol Neurol 2015;36:575-80. 10.1097/MAO. 0000000000000704.

[10]. De Ridder D, Vanneste S, Engineer ND, Kilgard MP. Safety and Efficacy of Vagus Nerve Stimulation Paired With Tones for the Treatment of Tinnitus : A Case Series. Neuromodulation 2014;17:170-9. 10.1111/ner.12127. [PubMed: 24255953]

[11]. Engineer CT, Hays SA, Kilgard MP. Vagus nerve stimulation as a potential adjuvant to behavioral therapy for autism and other neurodevelopmental disorders. J Neurodev Disord 2017 10.1186/ s11689-017-9203-z.

[12]. Khodaparast N, Hays SA, Sloan AM, Fayyaz T, Hulsey DR, Ii RLR, et al. Vagus Nerve Stimulation Delivered During Motor Rehabilitation Improves Recovery in a Rat Model of Stroke. Neurorehabilitative Neural Repair 2014;28:698-706. 10.1177/1545968314521006.

[13]. Kilgard MP, Rennaker RL, Alexander J, Dawson J. Vagus nerve stimulation paired with tactile training improved sensory function in a chronic stroke patient. NeuroRehabilitation n.d

[14]. Tyler R, Cacace A, Stocking C, Tarver B, Engineer N, Martin J, et al. Vagus Nerve Stimulation Paired with Tones for the Treatment of Tinnitus: A Prospective Randomized Double-blind Controlled Pilot Study in Humans. Sci Rep 2017 10.1038/s41598-017-12178-w.

[15]. Vanneste S, Martin J, Rennaker RL 2nd, Kilgard MP. Pairing sound with vagus nerve stimulation modulates cortical synchrony and phase coherence in tinnitus: An exploratory retrospective study. Sci Rep 2017 10.1038/s41598-017-17750-y.

[16]. Borland MS, Engineer CT, Vrana WA, Moreno NA, Engineer ND, Vanneste S, et al. The Interval Between VNS-Tone Pairings Determines the Extent of Cortical Map Plasticity. Neuroscience 2018;369:76-86. 10.1016/j.neuroscience.2017.11.004. [PubMed: 29129793]

[17]. Loerwald KW, Borland MS, Rennaker Ii RL, Hays SA, Kilgard MP. The interaction of pulse width and current intensity on the extent of cortical plasticity evoked by vagus nerve stimulation. Brain Stimul 2018;11:271-7. 10.1016/j.brs.2017.11.007. [PubMed: 29174302]

[18]. Heck C, Helmers SL, DeGiorgio CM. Vagus nerve stimulation therapy, epilepsy, and device parameters: scientific basis and recommendations for use. Neurology 2002;59:S31-7. 10.1212/ WNL.59.6_SUPPL_4.S31. [PubMed: 12270966]

[19]. Hulsey DR, Riley JR, Loerwald KW, Rennaker RL, Kilgard MP, Hays SA. Parametric characterization of neural activity in the locus coeruleus in response to vagus nerve stimulation. Exp Neurol 2017;289:21-30. 10.1016/j.expneurol.2016.12.005. [PubMed: 27988257]

[20]. Manta S, Dong J, Debonnel G, Blier P. Optimization of vagus nerve stimulation parameters using the firing activity of serotonin neurons in the rat dorsal raphe. Eur Neuropsychopharmacol 2009;19:250-5. 10.1016/j.euroneuro.2008.12.001. [PubMed: 19150228]

[21]. Chase MH, Nakamura Y, Clemente CD, Sterman MB. Afferent vagal stimulation: neurographic correlates of induced EEG synchronization and desynchronization. Brain Res 1967;5:236-49. 10.1016/0006-8993(67)90089-3. [PubMed: 6033149] 
[22]. Zabara J Inhibition of Experimental Seizures in Canines by Repetitive Vagal Stimulation. Epilepsia 1992;33:1005-12. 10.1111/j.1528-1157.1992.tb01751.x. [PubMed: 1464256]

[23]. Jiao J, Harreby KR, Sevcencu C, Jensen W. Optimal Vagus Nerve Stimulation Frequency for Suppression of Spike-and-Wave Seizures in Rats. Artif Organs 2016;40:E120-7. 10.1111/aor. 12669. [PubMed: 26713661]

[24]. Shetake JA, Engineer ND, Vrana WA, Wolf JT, Kilgard MP. Pairing tone trains with vagus nerve stimulation induces temporal plasticity in auditory cortex. Exp Neurol 2012;233:342-9. 10.1016/ j.expneurol.2011.10.026. [PubMed: 22079155]

[25]. Malow BA, Edwards J, Marzec M, Sagher O, Fromes G. Effects of vagus nerve stimulation on respiration during sleep. Neurology 2000;55:1450-4. [PubMed: 11094096]

[26]. Ben-menachem E Reviews Vagus-nerve stimulation for the treatment of epilepsy 2002;1:477-82.

[27]. Cunningham JT, Mifflin SW, Gould GG, Frazer A. Induction of c-Fos and DFosB Immunoreactivity in Rat Brain by Vagal Nerve Stimulation. Neuropsychopharmacology 2008;33:1884-95. 10.1038/sj.npp.1301570. [PubMed: 17957222]

[28]. Polley DB, Read HL, Storace DA, Merzenich MM. Multiparametric Auditory Receptive Field Organization Across Five Cortical Fields in the Albino Rat. J Neurophysiol 2007;97:3621-38. 10.1152/jn.01298.2006. [PubMed: 17376842]

[29]. Hulsey DR, Hays SA, Khodaparast N, Ruiz A, Das P, Rennaker RL, et al. Reorganization of Motor Cortex by Vagus Nerve Stimulation Requires Cholinergic Innervation. Brain Stimul 2016;9:174-81. 10.1016/j.brs.2015.12.007. [PubMed: 26822960]

[30]. Hays SA, Khodaparast N, Ruiz A, Sloan AM, Daniel R, Ii RLR, et al. The timing and amount of vagus nerve stimulation during rehabilitative training affect post-stroke recovery of forelimb strength. Neuroreport 2015;25:682-8. 10.1097/WNR.0000000000000154.The.

[31]. Hays SA, Rennaker RL, Kilgard MP. Targeting plasticity with vagus nerve stimulation to treat neurological disease. Prog Brain Res 2013;207 10.1016/B978-0-444-63327-9.00010-2.

[32]. Khodaparast N, Hays SA, Sloan AM, Hulsey DR, Ruiz A, Pantoja M, et al. Neurobiology of Disease Vagus nerve stimulation during rehabilitative training improves forelimb strength following ischemic stroke. Neurobiol Dis 2013;60:80-8. 10.1016/j.nbd.2013.08.002. [PubMed: 23954448]

[33]. Hays SA. Enhancing Rehabilitative Therapies with Vagus Nerve Stimulation. Neurotherapeutics 2016:382-94. 10.1007/s13311-015-0417-z. [PubMed: 26671658]

[34]. Pruitt DT, Schmid AN, Kim LJ, Abe CM, Trieu JL, Choua C, et al. Vagus Nerve Stimulation Delivered with Motor Training Enhances Recovery of Function after Traumatic Brain Injury 2015:871-9. 10.1089/neu.2015.3972.

[35]. He K, Huertas M, Hong SZ, Tie XX, Hell JW, Shouval H, et al. Distinct Eligibility Traces for LTP and LTD in Cortical Synapses. Neuron 2015;88 10.1016/j.neuron.2015.09.037.

[36]. Gu Z, Yakel JL. Timing-Dependent Septal Cholinergic Induction of Dynamic Hippocampal Synaptic Plasticity. Neuron 2011;71:155-65. 10.1016/j.neuron.2011.04.026. [PubMed: 21745645]

[37]. Seol GH, Ziburkus J, Huang S, Song L, Kim IT, Takamiya K, et al. Neuromodulators Control the Polarity of Spike-Timing-Dependent Synaptic Plasticity. Neuron 2007;55:919-29. 10.1016/ j.neuron.2007.08.013. [PubMed: 17880895]

[38]. Hassert DL, Miyashita T, Williams CL. The Effects of Peripheral Vagal Nerve Stimulation at a Memory-Modulating Intensity on Norepinephrine Output in the Basolateral Amygdala. Behav Neurosci 2004;118:79-88. 10.1037/0735-7044.118.1.79. [PubMed: 14979784]

[39]. Dorr AE, Debonnel G. Effect of Vagus Nerve Stimulation on Serotonergic and Noradrenergic Transmission. J Pharmacol Exp Ther 2006;318:890-8. 10.1124/jpet.106.104166.and. [PubMed: 16690723]

[40]. Nichols JA, Nichols AR, Smirnakis SM, Engineer ND, Kilgard MP, Atzori M. Vagus nerve stimulation modulates cortical synchrony and excitability through the activation of muscarinic receptors. Neuroscience 2011;189:207-14. 10.1016/j.neuroscience.2011.05.024. [PubMed: 21627982] 
[41]. Follesa P, Biggio F, Gorini G, Caria S, Talani G, Dazzi L, et al. Vagus nerve stimulation increases norepinephrine concentration and the gene expression of BDNF and bFGF in the rat brain. Brain Res 2007;79:28-34. 10.1016/j.brainres.2007.08.045.

[42]. Roosevelt RW, Smith DC, Clough RW, Jensen RA, Browning RA. Increased extracellular concentrations of norepinephrine in cortex and hippocampus following vagus nerve stimulation in the rat. Brain Res 2006;9:124-32. 10.1016/j.brainres.2006.08.048.

[43]. Feldmeyer D, Flores G, Concetta Miniaci M, Atzori M, Cuevas-Olguin R, Esquivel-Rendon E, et al. Locus Ceruleus Norepinephrine Release: A Central Regulator of CNS Spatio-Temporal Activation? 2016;8 10.3389/fnsyn.2016.00025.

[44]. Clark KB, Naritoku DK, Smith DC, Browning R a, Jensen R a. Enhanced recognition memory following vagus nerve stimulation in human subjects. Nat Neurosci 1999;2:94-8. 10.1038/4600. [PubMed: 10195186]

[45]. Clark KB, Smith DC, Hassert DL, Browning RA, Naritoku DK, Jensen RA. Posttraining Electrical Stimulation of Vagal Afferents with Concomitant Vagal Efferent Inactivation Enhances Memory Storage Processes in the Rat. Neurobiol Learn Mem 1998;70:364-73. [PubMed: 9774527]

[46]. Zuo Y, Smith DC, Jensen RA. Vagus nerve stimulation potentiates hippocampal LTP in freelymoving rats. Physiol Behanvior 2006:583-9. 10.1016/j.physbeh.2006.11.009.

[47]. Castoro MA, Yoo PB, Hincapie JG, Hamann JJ, Ruble SB, Wolf PD, et al. Excitation properties of the right cervical vagus nerve in adult dogs. Exp Neurol 2011;227:62-8. 10.1016/j.expneurol. 2010.09.011. [PubMed: 20851118]

[48]. Hays SA. Improving Stroke Rehabilitation with Vagus Nerve Stimulation Transl. Res. Stroke, Springer, Singapore; 2017, p. 503-15. 10.1007/978-981-10-5804-2_23.

[49]. Dawson J, Pierce D, Dixit A, Kimberley TJ, Robertson M, Tarver B, et al. Safety, Feasibility, and Efficacy of Vagus Nerve Stimulation Paired With Upper-Limb Rehabilitation After Ischemic Stroke. Stroke 2016;47:143-50. 10.1161/STROKEAHA.115.010477. [PubMed: 26645257]

[50]. Noble L, Gonzalez I, Meruva V, Callahan K, Belfort B, Ramanathan K, et al. Effects of vagus nerve stimulation on extinction of conditioned fear and post-traumatic stress disorder symptoms in rats. Transl Psychiatry 2017;7 10.1038/tp.2017.191. 


\section{Highlights}

- VNS paired with a sound strengthens the auditory cortex (A1) response to the sound.

- $\quad$ VNS-tone pairing directs A1 plasticity as an inverted-U function of VNS pulse rate.

- High temporal precision is necessary in VNS-tone pairing protocols.

- Insights derived from this work could lead to development of adjuvant therapies. 


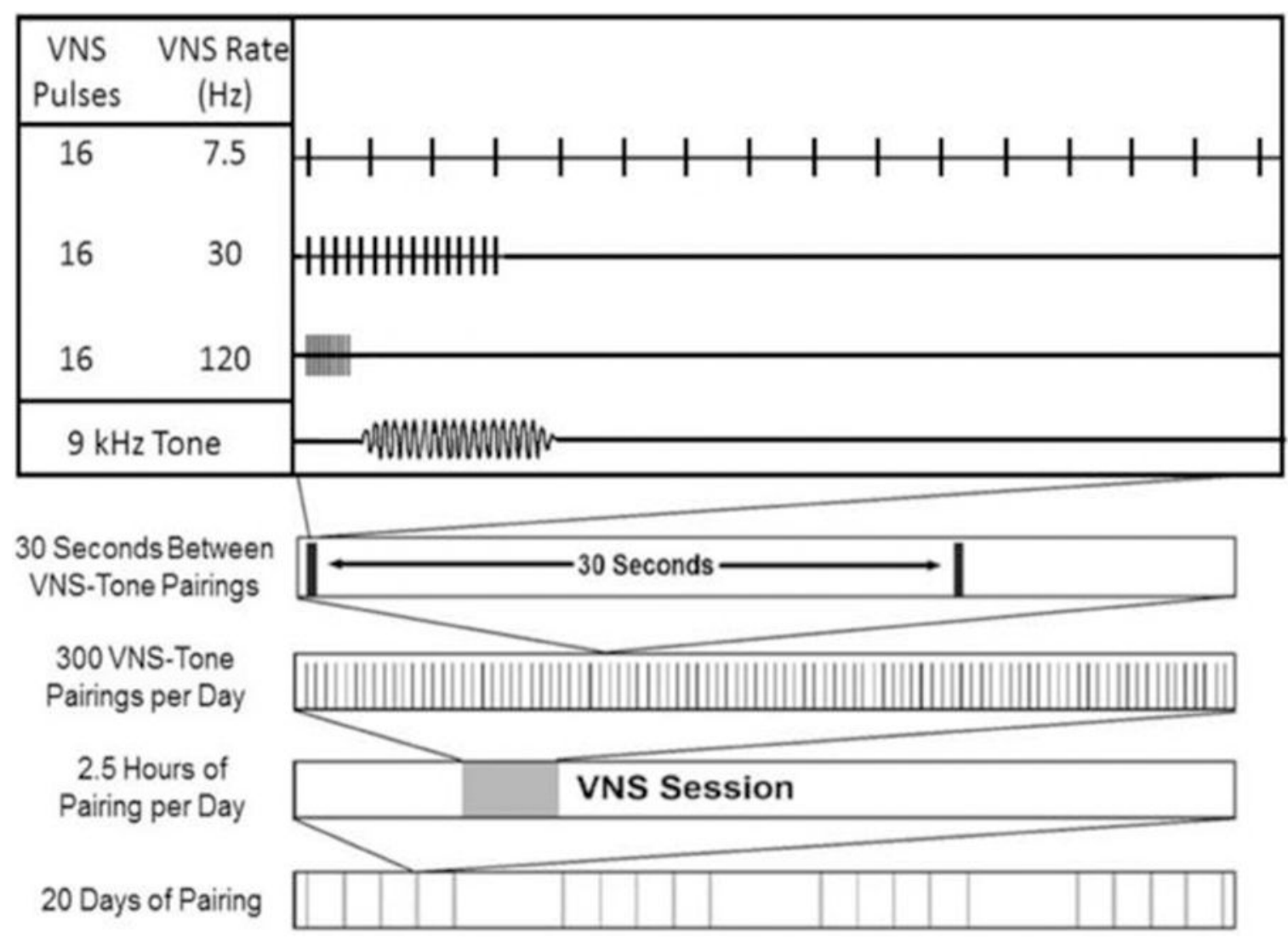

Figure 1.

Schematic diagram of the VNS-tone train pairing procedure. A train of biphasic pulses were delivered to the left vagus nerve via a cuff electrode. Each group received VNS at one of three different rates: $7.5 \mathrm{~Hz}(2 \mathrm{~s}), 30 \mathrm{~Hz}(0.5 \mathrm{~s})$, or $120 \mathrm{~Hz}(0.125 \mathrm{~s})$. Rats received VNS paired with a $9 \mathrm{kHz}$ tone every $30 \mathrm{~s}, 300$ times during each 2.5 hour session for 20 days. Cortical recordings were made 24 hours after the last pairing session. 


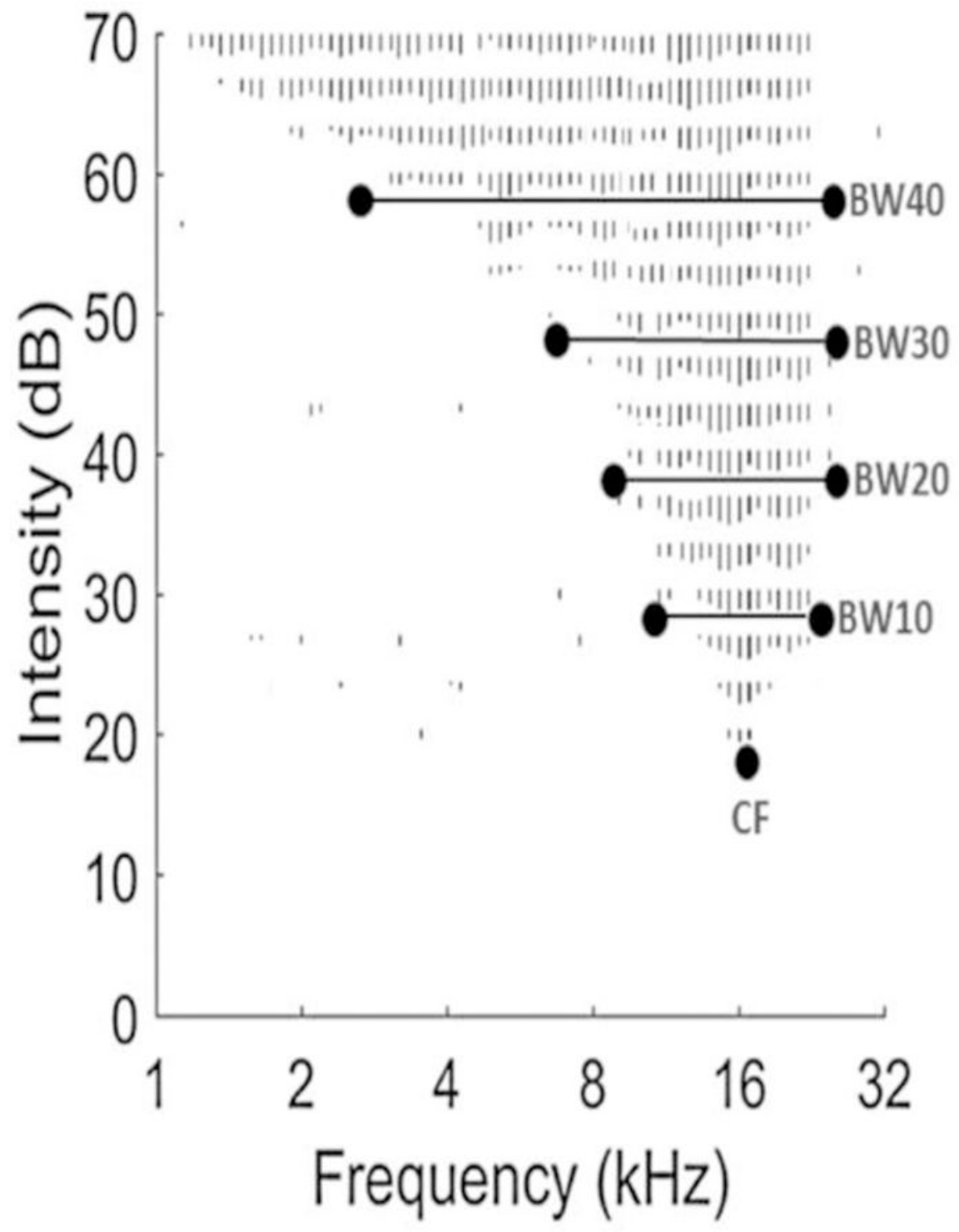

Figure 2.

Example tuning curve obtained from electrophysiological recordings in the primary auditory cortex (A1). The characteristic frequency (CF) is defined as the frequency with neuronal responses at the lowest intensity. Responses at higher intensity $(\mathrm{dB})$ levels are represented by bandwidth (BW). BW10, BW20, BW30, and BW 40 are responses at 10, 20, 30, and $40 \mathrm{~dB}$ above threshold intensity, respectively. 


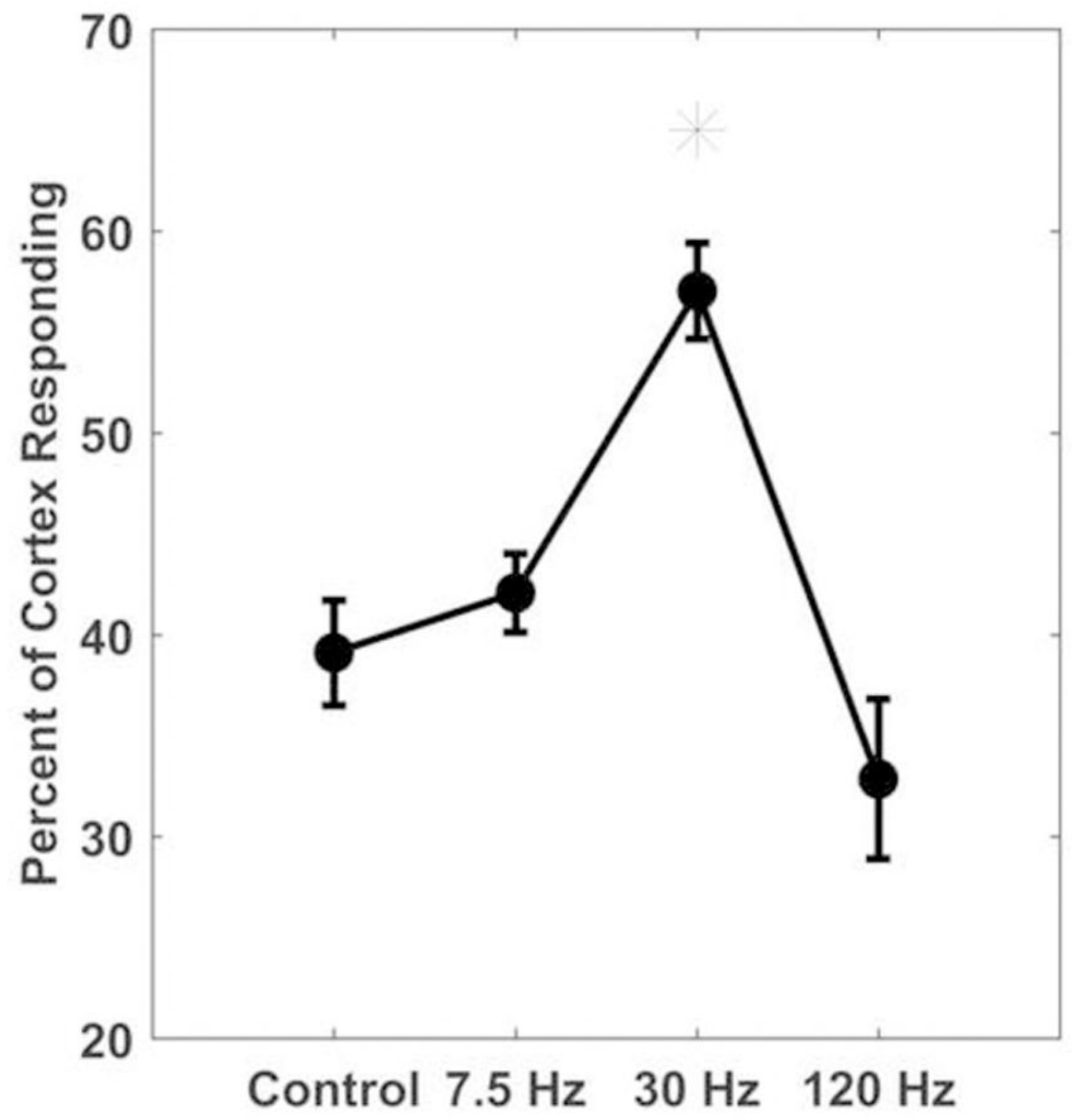

Figure 3.

VNS-tone pairing reorganizes the auditory cortex frequency map as a non-monotonic function of VNS rate. Moderate $(30 \mathrm{~Hz})$ rate VNS group exhibits a significant expansion of the percentage of A1 responding to $8-16 \mathrm{kHz}$ frequency tones at $50 \mathrm{~dB}$ SPL after 20 days of VNS tone-pairing $(\mathrm{p}<0.005)$. Low $(7.5 \mathrm{~Hz})$ and high $(120 \mathrm{~Hz})$ rate VNS groups do not yield a significant increase in neurons responding to $8-16 \mathrm{kHz}(\mathrm{p}>0.05)$. 


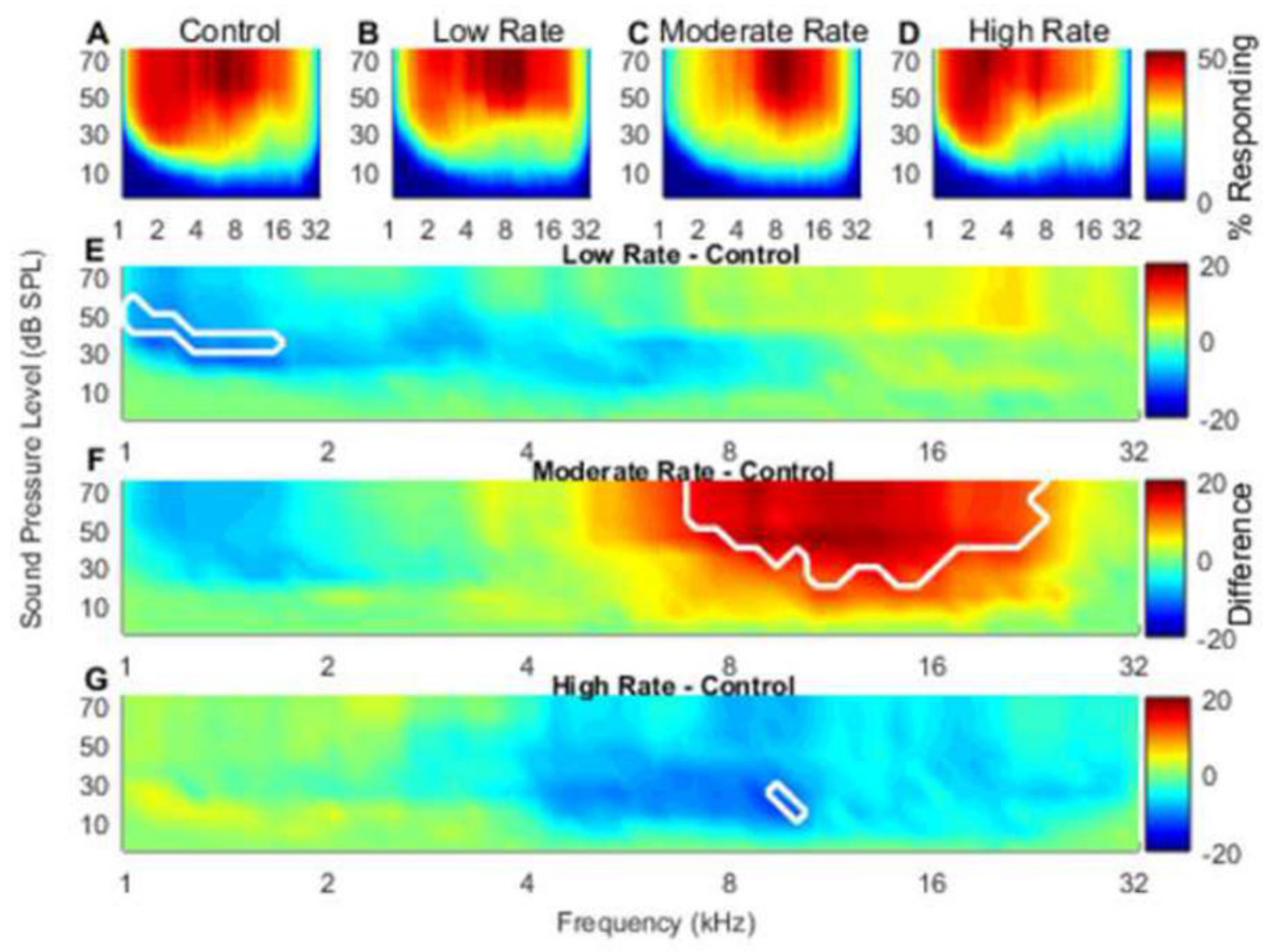

Figure 4.

Percent of A1 responding to each tone frequency intensity combination for naive control rats (A), low VNS rate $(7.5 \mathrm{~Hz})$ rats $(\mathrm{B})$, moderate rate $(30 \mathrm{~Hz})$ VNS rats $(\mathrm{C})$, and high rate $(120$ $\mathrm{Hz}$ ) VNS rats (D). The difference between the percent of A1 responding for low VNS rats and control rats reveals the range of tones that evoked a response in more neurons (red) or fewer neurons (blue) (E). The difference between the percent of A1 responding in moderate rate VNS rats and control rats $(\mathrm{F})$ and high rate VNS rats and control rats $(\mathrm{G})$ is also shown. Note that moderate intensity VNS-tone pairing alters the cortical response more than low and high rate VNS-tone pairing. White lines delineate the frequency intensity combinations which activate significantly more neurons after VNS pairing with a $9 \mathrm{kHz}$ tone $(\mathrm{p}<0.05)$. 


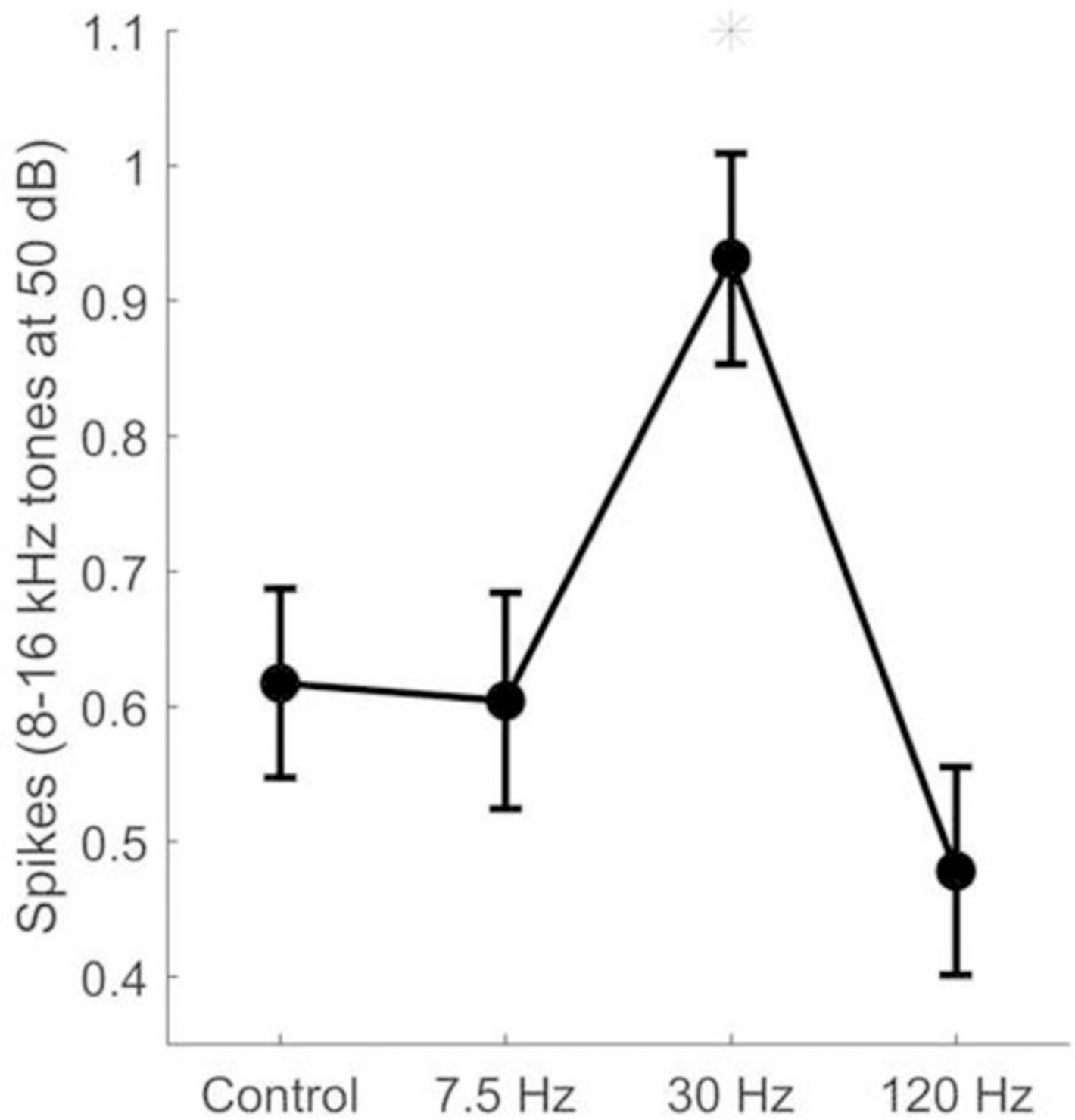

Figure 5.

A1 action potentials evoked by $8-16 \mathrm{kHz} 50 \mathrm{~dB}$ SPL tones significantly increases after animals receive moderate $(30 \mathrm{~Hz})$ VNS. This change is not observed with low $(7.5 \mathrm{~Hz})$ or high $(120 \mathrm{~Hz})$ rate VNS. 


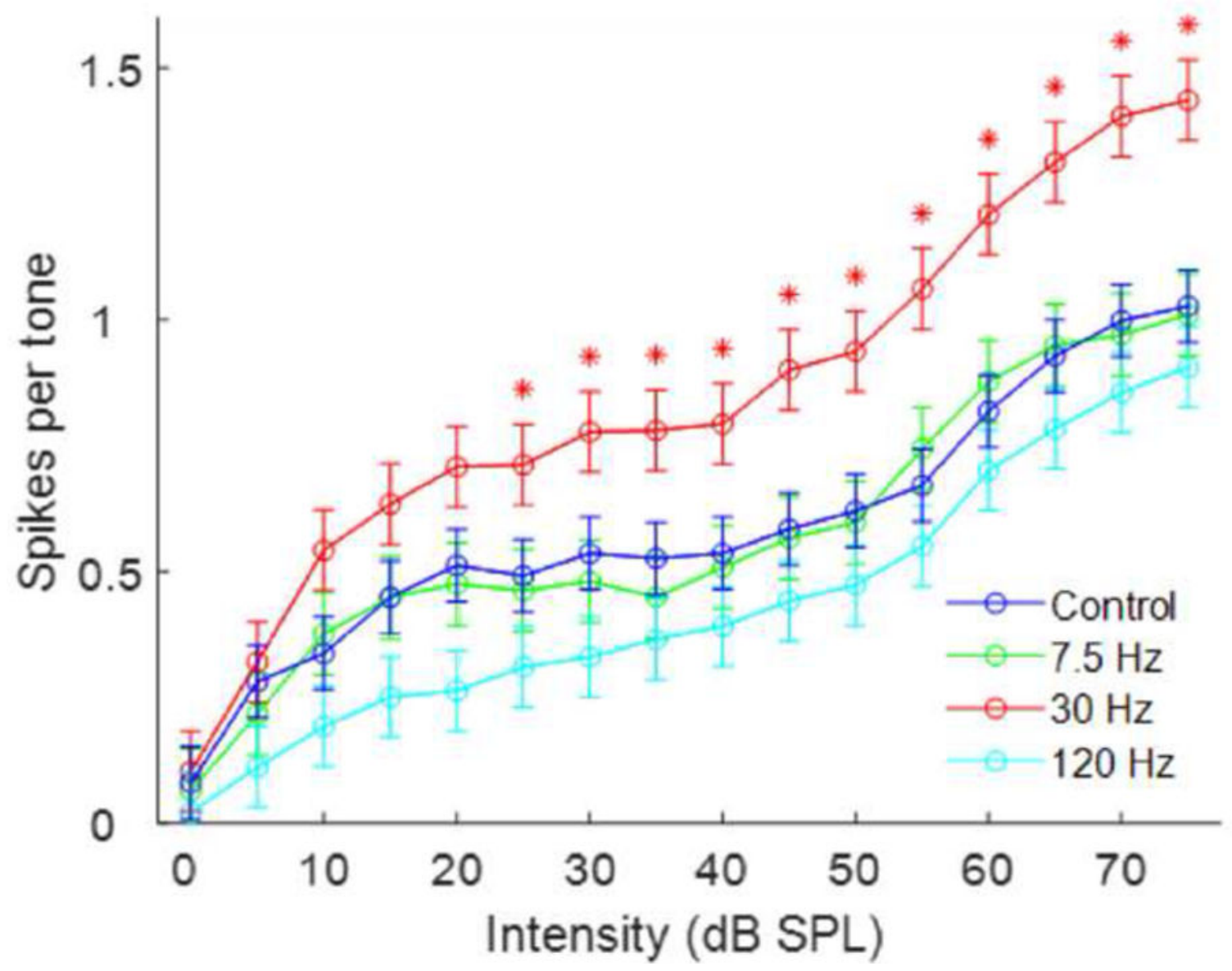

Figure 6.

Number of spikes evoked with increasing intensity in naive control, low rate VNS $(7.5 \mathrm{~Hz})$, moderate rate VNS $(30 \mathrm{~Hz})$, and high rate VNS $(120 \mathrm{~Hz})$ animals. Moderate rate VNS yields significantly stronger responses than control for intensities greater than $25 \mathrm{~dB}$ SPL $(\mathrm{p}<0.05)$. 